SESSION I

The Physician's Information NeED

\author{
Chairman: Professor G. E. MAWER
}

\title{
The general practitioner's information requirements in prescribing
}

\author{
D. W. Dingwall \\ M.B., Ch.B., M.R.C.G.P. \\ Glenwood Health Centre, Glenrothes, Fife
}

\begin{abstract}
Summary
A general practitioner's requirements can be described in terms of three levels. These three levels are described and discussed with reference to examples from the author's own experience.
\end{abstract}

THE author feels that information about drugs is required by the general practitioner at three levels:

Level I. He must have a reasonable knowledge of basic pharmacology and therapeutics so that the concepts of drug usage are understood.

Level II. In a given disease condition he must have knowledge of the drugs for use in that condition.

Level III. In a given patient he must have knowledge of how to choose the most suitable drug for that particular patient.

Knowledge is required at the first level by the general practitioner not only as a background to basic understanding of what he is about but also of what is regularly required in day-to-day situations. For example, if a child has conjunctivitis and otitis media, will an antibiotic for the ear infection be excreted in the lachrymal fluid or be sufficiently active in the conjunctiva to avoid the need for eye preparations as well?

When trying to decide whether a new penicillin preparation is worth trying or not, one has to have some understanding of the significance of the described data on the plasma levels, tissue levels and degree of protein binding. This sort of knowledge is particularly important to the general practitioner in his assessment of a new product.

All doctors must have had sufficient knowledge of pharmacology to pass exams at medical school or none of them would be a general practitioner now, but of course the memory can get rusty, advances in knowledge occur steadily and they are constantly in danger of basing their understanding on increasingly outmoded concepts. Unfortunately pharmacology is likely to have trouble competing with other more clinical topics for the attention of the busy general practitioner who probably has very limited time for reading anything.

Here, then, is a clearly definable need-for pharmacological advances, of relevance to general clinical problems, to be brought to the attention of the ordinary busy general practitioner in a way which highlights their relevance and promotes their assimilation into the doctor's background knowledge. It is not suggested that the general practitioner should make no effort at all to obtain such knowledge, or that he should be encouraged to sit back and wait for the most recent breakthrough to be spoon-fed to him. What is suggested is that, for the general practitioner-with the whole range of medical practice to cover-keeping up to date by reading scientific journals is a hopeless task. He is willing to learn, but can only do so if he has access to information which is selected for its relevance to his work.

There are many publications which do this in purely clinical subjects-the problem then is which of the many should one concentrate on? There may well be publications which do the same in clinical pharmacology, but the author is certainly not sure where to look for the answer to his earlier question about the effectiveness of systemic antibiotics in conjunctival infection.

It is not possible to start treating any patients with rheumatoid arthritis, for example, without knowledge of the drugs available for use in that 
condition. Therefore, at the second level it is an essential part of a practitioner's continuing education that he maintains his knowledge of the suitable drugs and adjusts it in the light of new findings as they occur.

For rheumatoid arthritics in general what is the place of prednisolone by mouth? Is ACTH by injection better? Ibuprofen at the originally suggested dosage may be undramatic in its effectiveness, but is it, in fact, much more effective in the higher doses now being suggested? These are the sort of questions, relevant to the whole group of rheumatoid arthritic patients, to which general practitioners require answers if they are to be sure of giving their patients the best chance of the best treatment.

In trying to maintain some rational prescribing policy in the face of ever-increasing masses of conflicting claims and counter-claims about the relative effectiveness of the different drugs for particular conditions, many general practitioners no doubt resort to the two down-to-earth, commonsense aphorisms-'never be the first to use a new drug or the last to stop using an old one' and 'given a wide choice of drugs, stick to a few and get to know them well'. While the basic wisdom of these is not disputed, they unfortunately do not cover a variety of real problems that are constantly met.

Considering the first of the above statementsone is seldom in a position to be the first, or even the one-hundred-and-first, to use any new drug, but one may still be faced with the question-is this new drug really better than the old favourites? If one is cautious and does not start using it, is it possible that one's patients are being denied the chance of significant improvement in their treatment? It is known that most 'wonder drugs' turn out to be a bit disappointing in the long run, but can one really be sure that this one, this time, isn't the exception as Intal was in childhood asthma? Of course, with all the dangers of drug usage well in mind nowadays, one must not be rash, but one may fall far short of optimum effectiveness if one is over-cautious instead.

What is needed is easily accessible, reliable, unbiased information about new drugs based on well designed drug trials. Promotional literature from drug firms can be very valuable in drawing attention to a new drug, and is certainly easily accessible, and it may be reliable and unbiased, but very few general practitioners would feel confident of being such about reliability and lack of bias. It may often be possible to detect the flaws in unsound promotion but few would feel expert enough to be certain that apparently sound information is indeed as good as it looks. How many GPs know a completely flawless drug trial when they see one?

What is now needed, then, is easily accessible, reliable information about new drugs, based on well designed trials, presented in a convincing way by someone who knows what he is talking about, who $\stackrel{\mathbb{Q}}{\complement}$ knows about drug trial design, and who has de- $c$. veloped considerable expertise in critical assessment $\overrightarrow{\vec{F}}$ of drug research. Such a presentation would really $\stackrel{?}{\rightarrow}$ be able to help physicians decide when to give new drugs a trial in their patients and when to leave them in the hands of researchers for a bit longer.

Considering the second of the two aphorisms $\stackrel{\mathbb{D}}{\Omega}$ above - the same sort of arguments apply when one \& is faced with a wide choice of drugs. The physician $\vec{\circ}$ intends to prescribe only a few of the many diuretics, only a few of the very many tranquillizers and anti- $\vec{\omega}$ depressants. This of course, makes a lot of sense if $\frac{\Omega}{8}$ they are all much the same in each group, but it 6 makes much less sense for him to get to know one 3 . well, if another were in fact a generally better drug. 이

Much more in the way of reliable comparative 0 data is needed-not just between, say, tricyclic $\omega$ antidepressants and mono-amine oxidase inhibitors, of but between individual drugs in the tricyclic group. 0 This would allow each tricyclic compound to be $\frac{}{J}$ compared in relevant ways with all the others in that $\vec{c}$ group. In short, what is needed is a regularly up- $\mathbb{D}$ dated 'Which?' for drugs.

At the third level, the 'crunch' point in generel 3 practice-prescribing is reached. All the carefunl build-up of pharmacological background, am $\overrightarrow{0}$ detailed comparative analysis to determine the best antidepressant available, is to no avail if the patiet. comes back for review and says 'Well, they may be good pills doctor, but I wanted to sleep at night not during the day'.

Of course, it is accepted that there is often no substitute for trial and error until the most suitable $\stackrel{\AA}{\complement}$ preparation for a particular patient is found, but is $\overrightarrow{\hat{0}}$ there no way to improve the chances of selecting the 3 right drug first time? Could dosage recommendations not be more explicit about the best way of reaching the optimum dose of a particular anti- $\overline{-}$ depressant in a 75-year-old lady? One wishes to 3 . relieve her depression rapidly, but if the dose is pushed up too quickly, the side effects may result. in her losing confidence in the drug altogether. Sheo would then request a change which produces delay while the same process is repeated with a different? preparation.

The author knows there is no magic answer and을. that experience and good judgment are the main requirements. However, is there no way of drawing on the experience and judgement of others who may have seen more cases for example? It is possible, of course, to telephone consultant colleagues, but they are busy too and may be difficult to find quicklyøo Also one may be just a little reluctant regularly toळ expose one's ignorance to colleagues with whom one? has a close working relationship. What is needed 
are colleagues who are readily available, who are willing to act as fact-finding agents, and who have access to a wide range of experience and knowledge. With the help of such colleagues one could feel much more confident in dealing with difficult therapeutic problems, could develop rational dosage regimes more easily, and could work out best drug choices for individual patients.

None of the books to hand in the surgery was of much help when a severe rheumatoid arthritic was discharged from hospital, after a brisk haematemesis from a duodenal ulcer, on a regime of light diet and aluminium hydroxide gel (Aludrox). What should she take for her joints? she wanted to know. The author knew that if he didn't restart the drugs for her rheumatoid arthritis soon, then life for her would become continually dominated by pain, Yet, with the wrong drugs, life might cease altogether if severe haemorrhage occurred again. Which drug, of the many available, would most likely achieve the balance between maximum anti-rheumatic and analgesic effect and minimum gastro-intestinal upset? To answer that question effectively, access was needed, not to text books, journals or review articles, but to a pool of experience of that rather limited and uncommon situation.

In conclusion, even though none of the needs described in this paper were to be met, general practitioners would still manage to treat their patients, as they have done for generations. If all of them could be met, however, these powerful tools given by the pharmaceutical industry could be used to far greater effectiveness and lesser risk-surely very laudable aims in improving care of patients. 